\title{
Imagery, memory, and size-distance invariance
}

\author{
TIMOTHY L. HUBBARD, DARREN KALL, and JOHN C. BAIRD \\ Dartmouth College, Hanover, New Hampshire
}

\begin{abstract}
The size-distance invariance hypothesis (SDIH) was examined for remembered and imaged stimuli. In Experiment 1, subjects gave remembered and imaged distances of familiar objects and imaged distance of nondescript rods. The relationship between stated size and distance is more adequately described by power functions with exponents less than 1 than by the more restricted SDIH (exponent of 1). In Experiment 2, subjects gave distance estimates to recalled and imaged familiar objects and described the visual context in which each object was situated. A different group then sorted the contexts into categories based on general similarity. There were no significant differences between distance estimates based on memory and those based on imagery, and the visual contexts were not sorted according to whether they were generated in the memory or in the imagery conditions. In Experiment 3, subjects estimated the distances to objects in an outdoor setting. A linear relationship was found between estimated and physical distance, suggesting that the lower exponents obtained in Experiments 1 and 2 were not artifacts of the distance judgment procedure.
\end{abstract}

In the course of examining the cognitive resources utilized in visual imagery, Kosslyn (1978) measured the maximum extent of the visual field used in visual imagery. He found a linear relationship between the stated size of a familiar object and the minimum distance at which the entire object could be portrayed in an image. This distance, the point at which an imaged object overflowed, was taken to delineate the maximum visual angle (boundaries) of the "mind's eye." Such a result suggests that the "imaged" relationship between known size and distance agrees with the size-distance invariance hypothesis (SDIH): for a fixed visual angle $(\phi)$, the ratio of perceived size $(S)$ to perceived distance $(D)$ of an object in real space is constant:

$$
\begin{aligned}
& \tan \phi=S / D \\
& D=S / \tan \phi .
\end{aligned}
$$

The visual angle at overflow, $\phi$ in Equation 1, is taken to be a constant for each subject and each class of stimuli. (Note that if $S$ and $D$ are specified, this is a one-parameter model, and that in Equation $1 \mathrm{~b}$ the $y$-intercept of the function is zero.) The maximum size of the imaged visual angle is not constant, however, as shown by the wide range of values $\left(13^{\circ}\right.$ to $\left.50^{\circ}\right)$ that Kosslyn (1978) obtained for different classes of objects. The SDIH appears to be satisfied, but different linear functions (slopes in Equation 1) hold for different categories of objects. More importantly, the visual angle calculated along the best fitting function for a particular class does not remain constant when larger

\footnotetext{
The authors thank Jamshed Bharucha, Julian Hochberg, Margaret Jean Intons-Peterson, and two anonymous reviewers for helpful comments on an earlier draft of the manuscript. Correspondence should be addressed to John C. Baird, Department of Psychology, Dartmouth College, Hanover, NH 03755.
}

stimuli are excluded, implying that different functions hold for different object sizes.

The connection between perceived size and distance has been a prime topic of research in visual perception for over a century (for reviews, see Baird, 1970; Epstein, Park, \& Casey, 1961; Sedgwick, 1986). If in total darkness a familiar target is illuminated, a subject is able to accurately estimate its distance. The key findings were first reported by Hastorf (1950) and Ittelson (1951); subsequently verified by Baird (1963), Coltheart (1969a, 1969b), and Ono (1969); and reaffirmed more recently by Fitzpatrick, Pasnak, and Tyer (1982). Similarly, if the distance to an illuminated target presented in total darkness is known, a subject can accurately state its metric size (Coltheart, 1970; Park \& Michaelson, 1974).

In typical perception experiments, a target is presented. Subjects are thus given knowledge of two of the variables in Equation 1 and are able to estimate the value of the third variable. In imagery experiments, however, a target is not presented. Subjects are given knowledge about only one of the variables in Equation 1 (stated metric size) and must use this information to estimate metric distance. Since subjects are given knowledge of only one of the three variables in Equation 1, they are unable to arrive at a unique solution. When faced with this task, subjects may create an image of the named object and read the resultant values of at least one of the other variables off their image. If properties of metric scale are represented in the memory that underlies image generation, then a lawful relationship between stated size and portrayed distance should exist prior to any transformation of the created image. If properties of metric scale are not represented in the memory that underlies image generation, then there is no reason to postulate a unique relationship between stated size and portrayed distance prior to any transformation. In this latter case, active size-distance scaling of 
the imaged objects would be necessary in order for the SDIH to be satisfied.

Kosslyn (1980) briefly reported studies of the "spontaneous distances" at which different animals are imaged. He reported that the relationship is linear, but, again, the angle calculated from the best fitting function is not constant; images of larger animals and images of smaller animals subtend different visual angles. The apparent decrease of imaged visual angle with increasing stated physical size suggests, however, that the relationship between stated size and imaged distance in an untransformed image may be better described by a nonlinear function.

The primary issue in the following experiments is whether, despite the inadequacy of information available for uniquely solving Equation 1, there is a constant ratio (visual angle) between the stated metric size of the target and the portrayed distance of the target estimated by the subject in untransformed images. If the SDIH is valid under these circumstances, then Kosslyn's (1980) finding of a linear relationship between size and imaged distance for both spontaneous and overflow conditions may be due merely to the ability of subjects to recall the distances at which objects of different physical sizes are usually seen and stored in memory. A secondary issue in the present experiments was whether the performance of subjects instructed to image would differ from the performance of subjects instructed to remember. If there is no difference between the psychophysical functions obtained under imagery and memory instructions, then claims that the relationship between stated size and imaged distance is due to properties of an imagery medium would clearly be weakened since the same data could be described as due to nonimaginal processes of memory. If, however, there is a difference between the psychophysical functions obtained under imagery and memory instructions, this would support the necessity of imagery transformations.

\section{EXPERIMENT 1}

In this experiment, subjects were presented with the names and sizes of familiar objects of known metric sizes and were told to estimate the objects' imaged or remembered distances. We assumed that the objects are frequently encountered in everyday life, and that subjects would be able to image or recall exemplars. Since different objects are typically experienced at different distances in the world outside the laboratory, it is possible that memory of the distance at which an object is typically seen affects the distance that is portrayed in the image. We included a control condition in which subjects estimated the distances portrayed in images of nondescript rods. The metric sizes of the rods were given by the experimenter. It is doubtful that many rods of the sizes given were ever seen by subjects in the past, so this condition sidestepped the direct influence of past learning experiences. The rods, therefore, should have had no previous perceived dis- tances associated with them, and thus were expected to yield less variability than the familiar objects. If metric size is indeed the critical parameter of imaged distance, then familiar objects and unfamiliar rods would be related to imaged and remembered distance by approximately similar functions.

\section{Method}

Subjects. The participants were 60 Dartmouth College undergraduates who received extra course credit in their introductory psychology courses. They were randomly assigned to either the memory or the imagery group.

Materials. The stimuli consisted of a set of 21 names of familiar objects (see Table 1) and a set of 14 sizes of nondescript rods. The sizes of the rods were matched to the stated sizes of the familiar objects. Kosslyn (1980) found that subjects tend to use the longest metric size (other than the diagonal) to guide their imagery judgments; thus, all our stimuli were described by the single largest extent (length, width, or height). The actual sizes used were determined by informal agreement among 4 graduate students.

General procedure. All subjects sat in a windowless classroom that contained a chalkboard and some chairs situated around several large tables. Each stimulus name and size was typed on a separate sheet of paper; these sheets were collected into booklets in which the subjects entered their judgments.

Imagery task. Each booklet in the imagery task was divided into two sections: one listed the names and sizes of the 21 familiar objects, and the other listed the sizes of the 14 rods. Half of each group received the section containing the familiar objects first; the other half received the list of rods first. For each subject, the stimuli were randomly ordered within a section. Several anecdotal examples of visual imagery were given before the subjects' attention was directed to the chalkboard on which the following instructions were printed:

1. Read the name and size of the object. 2. Close your eyes. 3. Imagine the object. Use your first clear image and consider each image independently of the others. 4 . Write in your booklet how far away (in feet and inches) the object was from your head when you imagined it. 5 . Wait for my pace command before going on to the next object.

The subjects closed their eyes to reduce the possibility that current visual input would interfere with imagery, as has been implicated in previous studies (e.g., Farah, 1985; Segal \& Fusella, 1970). The subjects were instructed to use the rule of "first clear image," because in pilot studies individuals often reported having many images of an object, thus being forced to choose among them when rendering a judgment. Thirty seconds were allowed for each judgment.

Memory task. Each booklet in the memory task contained one section that listed the names and sizes of the 21 familiar objects; booklets did not include the unfamiliar rods, since "remembering" stimuli that have never been seen before is impossible. For each subject, the stimuli were randomly ordered. The experimenter's introductory remarks made no mention of mental or visual imagery, or of a "first clear image." The experiment was introduced as one concerned with "remembered familiar distances." The following instructions were printed on the chalkboard:

1. Read the name and size of the object. 2 . When you usually see this object, how far away from your head (in feet and inches) is it? 3. Write this distance in your booklet. 4 . Wait for my pace command before going on to the next object.

The general procedure followed that of the imagery task, except that the subjects were not told to close their eyes, since having them 
close their eyes might lead some to think that imagery was a demand of the task.

\section{Results and Discussion}

Intersubject variability was substantial. Table 1 lists the median distance estimate for each familiar object in the imagery and memory conditions. These data deviate significantly from linearity, and, in particular, the $y$ intercepts of Equation lb ( -13.47 for memory; 1.76 for imagery) are not zero. The SDIH is clearly not appropriate. A power function in which the overall relationship between distance and size is nonlinear (exponent less than 1) is more appropriate:

$$
\begin{gathered}
D=\lambda S^{\gamma} \\
\log D=\gamma \log S+\log \lambda .
\end{gathered}
$$

A least-squares fit of the data on logarithmic axes (Equation $2 \mathrm{~b}$ ) yielded a slope (exponent) of $\gamma=.59$ with a $y$ intercept of .52 for imagery $\left(r^{2}=.67\right)$. For memory, a least-squares fit yielded a slope of $\gamma=.57$ and a $y$-intercept of $.75\left(r^{2}=.40\right)$. If the SDIH were an appropriate model, the slope $(\gamma)$ of Equation $2 b$ should be close to 1; however, the slopes of both the imagery and the memory functions are considerably less than 1, supporting the earlier claim that the SDIH is clearly inappropriate.

Although these functions suggest a positive relationship between judged distance and size for familiar objects, the fits are relatively poor for both conditions. This variability may be related to past exposures to the objects in natural settings. Certain objects are commonly experienced within a restricted range of distances, and this range need not be a function solely of object size. For example, a bird's nest is a relatively small object that is seldom seen up close, whereas a refrigerator is a relatively large object that is seldom encountered outside the close confines of a kitchen. If these objects were imaged or recalled at their familiar distances, an underlying monotonic relationship between judged distance and object size would be distorted. Several of the stimuli were chosen to purposely highlight this potential effect of familiarity. The five stimulus persons, representing different social roles in society, might be expected in everyday experience to be seen at different distances. As one might predict on this basis, the actor, teacher, and policeman were imaged (mean of medians $=11.33 \mathrm{ft}$ ) and recalled $(21.66 \mathrm{ft}$ ) farther away than the store clerk and friend $(3.38 \mathrm{ft}$ and $3.00 \mathrm{ft}$, respectively).

It is well established in psychophysics that variability increases with stimulus intensity or with the intensity separation between a stimulus and a standard (Baird, 1970; Baird, Green, \& Luce, 1980; Baird \& Noma, 1978). If imaged and remembered distances follow such a pattern, then this would be consistent with the view that these processes share at least some cognitive processes or mechanisms (see Finke \& Shepard, 1986; Shepard \& Podgorny, 1978). The relevant data are shown in Tables 1 and 2; judgment variability is linearly related to the median for imagery $\left(r^{2}=.98\right)$ and memory $\left(r^{2}=.99\right)$, supporting the view that imagery and memory are functionally similar to perception. Furthermore, IQI for imagery is correlated with IQI for memory $(r=.96)$, suggesting functional similarities between memory and imagery. This increase in variability with increasing intensity is also con-

Table 1

Median Distances (in feet) and One-Half Interquartile

\begin{tabular}{|c|c|c|c|c|c|}
\hline \multirow[b]{2}{*}{ Object } & \multirow[b]{2}{*}{ Size } & \multicolumn{2}{|c|}{ Memory Condition } & \multicolumn{2}{|c|}{ Imagery Condition } \\
\hline & & Median & $\mathrm{IQ} \mathbf{l} / 2$ & Meciian & $\mathrm{IQI} / 2$ \\
\hline Coin & 1 in. & 1.50 & .38 & .75 & .25 \\
\hline Bird's nest & $6 \mathrm{in.}$ & 20.00 & 5.85 & 3.50 & 2.25 \\
\hline Telephone & $10 \mathrm{in.}$ & 2.75 & 1.75 & 2.00 & .50 \\
\hline Shoe & $11 \mathrm{in.}$ & 5.50 & .32 & 2.00 & 1.13 \\
\hline Street lamp & $12 \mathrm{in.}$ & 22.50 & 6.25 & 15.00 & 4.75 \\
\hline Dog & $18 \mathrm{in.}$ & 5.00 & .75 & 4.00 & .63 \\
\hline Crow & $18 \mathrm{in.}$ & 50.00 & 13.75 & 12.00 & 6.75 \\
\hline Television & 24 in. & 8.00 & 1.00 & 5.00 & 1.13 \\
\hline Chair & 36 in. & 2.25 & .57 & 3.00 & .50 \\
\hline Flag & 36 in. & 32.50 & 8.75 & 14.00 & 3.75 \\
\hline Friend & 70 in. & 3.00 & .69 & 2.75 & .63 \\
\hline Teacher & 70 in. & 10.00 & 3.13 & 10.00 & 2.25 \\
\hline Store clerk & 70 in. & 3.00 & .57 & 4.00 & .75 \\
\hline Actor & 70 in. & 40.00 & 6.25 & 16.00 & 8.00 \\
\hline Policeman & 70 in. & 15.00 & 3.75 & 8.00 & 4.00 \\
\hline Refrigerator & 84 in. & 2.50 & .59 & 4.00 & .75 \\
\hline Piano & $10 \mathrm{ft}$ & 6.50 & 1.88 & 6.00 & 1.75 \\
\hline Car & $10 \mathrm{ft}$ & 10.00 & 5.57 & 10.00 & 2.50 \\
\hline Boat & $50 \mathrm{ft}$ & 100.00 & 142.50 & 30.00 & 20.00 \\
\hline Building & $50 \mathrm{ft}$ & 50.00 & 40.50 & 50.00 & 20.00 \\
\hline Airplane & $230 \mathrm{ft}$ & 1000.00 & 3712.50 & 240.00 & 85.00 \\
\hline
\end{tabular}
Intervals (IQI/2) in Experiment 1 
Table 2

Median Distances (in feet) and One-half Interquartile Intervals (IQI/2) for Imaged Rods in Experiment 1

\begin{tabular}{rrr}
\hline Size & Median & IQI/2 \\
\hline $1 \mathrm{in.}$ & .75 & .13 \\
$6 \mathrm{in.}$ & 1.00 & .32 \\
$10 \mathrm{in.}$ & 1.50 & .57 \\
$11 \mathrm{in.}$ & 2.00 & .50 \\
$12 \mathrm{in.}$ & 1.50 & .38 \\
$18 \mathrm{in.}$ & 2.00 & .75 \\
$1.5 \mathrm{ft}$ & 2.25 & .38 \\
$24 \mathrm{in.}$ & 3.00 & .38 \\
$36 \mathrm{in.}$ & 3.50 & .50 \\
$70 \mathrm{in.}$ & 4.00 & 1.00 \\
$7 \mathrm{ft}$ & 5.50 & 1.50 \\
$10 \mathrm{ft}$ & 7.00 & 2.50 \\
$50 \mathrm{ft}$ & 20.00 & 5.00 \\
$230 \mathrm{ft}$ & 70.00 & 57.50 \\
\hline
\end{tabular}

sistent with the results of Weber and Malmstrom (1979), who found larger variability of imaged visual angles for words imaged at subjectively larger sizes.

To further test the role of the specific stimuli, we replicated the familiar object condition of Experiment 1 on a new group of 60 subjects. The cross-experiment correlations for median imagery and memory distances were substantial ( $r=.96$ and $r=.99$, respectively), consistent with the view that past experience with objects in the natural environment influences imaged or remembered distance.

The stimulus rods were never experienced before, so they offer a purer test of the relationship between metric size and portrayed distance. Table 2 presents the median distance and interquartile intervals of the rods as a function of object size. Least-squares regression on logarithmic axes yielded a slope of $\gamma=.60$ and a $y$-intercept of $.26\left(r^{2}=.96\right)$. Although the slope is quite similar to those obtained with imagery and memory for familiar objects, the goodness of fit is far superior.

The relationship between the metric size and the imaged distance is such that the visual angle occupied by the imaged object changes as the portrayed distance in the image changes. Therefore, metric and distance information inherent in untransformed images (or nonimaginal memories) cannot alone account for the linear overflow functions obtained by Kosslyn $(1978,1980)$; rather, some additional form of image transformation or memory sampling must occur in order for the size-distance scaling required by the SDIH to take place. These findings are consistent with the notion that subsequent size-distance scaling could result in a linear relationship between stated size and overflow distance, but are clearly at odds with Kosslyn's claim of a linear function relating stated size and spontaneous imaged distance.

\section{EXPERIMENT 2}

In this experiment, we further explored the relationship between imagery and inemory instructions by comparing the contexts that are portrayed in memories and images of familiar objects. When a subject remembers or images a familiar object, that object may be remembered or imaged in a specific setting or surrounding that is typical of the subject's experience with the object. For example, the surrounding context of an imaged car might be of a garage, a roadway, or a parking lot. If memories and images are based upon similar cognitive resources, then these accompanying contexts should be very similar; that is, the same familiar object should yield the same context whether it is remembered or imaged.

\section{Method}

Subjects. Thirty-five Dartmouth College undergraduates participated for extra course credit. They were randomly assigned into groups of 10,10, and 15. The two groups of 10 subjects served as the memory and imagery groups, and the remaining subjects participated in a sorting group.

Materials. The stimuli were 18 common objects of a wide range of sizes and shapes (see Table 3). The name of each object and its size on the longest axis were listed on individual $4 \times 6$ unlined file cards. The stated sizes of the objects were determined by taking the mean of size estimates given by 20 undergraduates who did not participate in the present experiment. Each subject in the imagery or memory conditions was given a stack of 18 cards in a different random order. For the sorting group, the materials consisted of 30 file cards that described visual contexts that had been generated by the imagery and memory groups.

General procedure. The subjects were run in subgroups of 5, but each subject worked individually. They were instructed to proceed through the stack of cards one object at a time. The subjects in both groups were allotted $1 \mathrm{~min}$ per card and were verbally paced by the experimenter.

Imagery task. The subjects read the name and size of each object and formed a mental picture of it. They were instructed to use their first clear picture and then write on the file card the distance (in feet and inches) and the context (setting) information portrayed in their image.

Memory task. The subjects read the name and size of each object and remembered an occasion when they had seen that object. They were encouraged to use their most vivid memory, but if they had never seen the object, they were to write "not seen"' on the file card. No distinction was drawn between the most recent and the most frequent episode. The subjects wrote on the file card both the distance (in feet and inches) of the recalled object from their

Table 3

Stimuli for Experiment 2

\begin{tabular}{lc}
\hline \multicolumn{1}{c}{ Object } & \multicolumn{1}{c}{ Size } \\
\hline Coin & 1 in. wide \\
Coffee mug & 4 in. tall \\
Dollar bill & 6 in. long \\
Toothbrush & 6 in. long \\
Chalk eraser & 6 in. long \\
Soda can & 6 in. high \\
Pencil & 8 in. long \\
Beer bottle & 9 in. high \\
Dinner plate & 10 in. wide \\
Football & 12 in. long \\
License plate & 12 in. long \\
House cat & 12 in. high \\
Rooster & 18 in. high \\
Stop sign & $2 \mathrm{ft}$ wide \\
Cow & $4.5 \mathrm{ft} \mathrm{high}$ \\
Teacher & $5 \mathrm{ft}, 10$ in. tall \\
Policeman & $6 \mathrm{ft}$ tall \\
Refrigerator & $6 \mathrm{ft}$ tall \\
\hline
\end{tabular}


memories and the context (physical setting) in which the object was situated.

Sorting task. The memory and imagery groups together made a total of 360 judgments concerning distance and physical context. These contexts were then typed onto individual slips of paper and glued to new file cards. Each subject in the sorting group was given a stratified random sample of 30 contexts. Each sample contained 15 contexts generated by the imagery group and 15 contexts generated by the memory group. The subjects were instructed to sort the contexts into as many stacks as they wanted and to use whatever criteria for similarity that they deemed relevant. We did not specify for subjects to sort into "imagery" and "memory" stacks because we wanted to see if that grouping would emerge spontaneously, and because we wanted to avoid giving them specific examples of imagery and memory contexts. After sorting all 30 objects, they wrote down the criteria they felt they had used.

\section{Results and Discussion}

A median distance was computed for each object in the imagery and memory groups. Figure 1 shows imaged distance (top panel) and remembered distance (bottom panel) as functions of object size. Least-squares regression on logarithmic axes revealed a slope (exponent) of $\gamma=.55$ and a $y$-intercept of .60 for the imagery condition $\left(r^{2}=.66\right)$. For the memory condition, the slope (exponent) was $\gamma=.47$, with a $y$-intercept of $.71\left(r^{2}=.30\right)$. These values are similar to those obtained in Experiment 1. Distance estimates for the memory and imagery conditions were highly correlated $(r=.88)[t(16)=7.49, p<.001]$. A least-squares solution of this relation, graphed in Figure 2, reveals a slope of .70 and an intercept of .26.

A two-way analysis of variance examined the influence of instructions (imagery or memory) and object size on
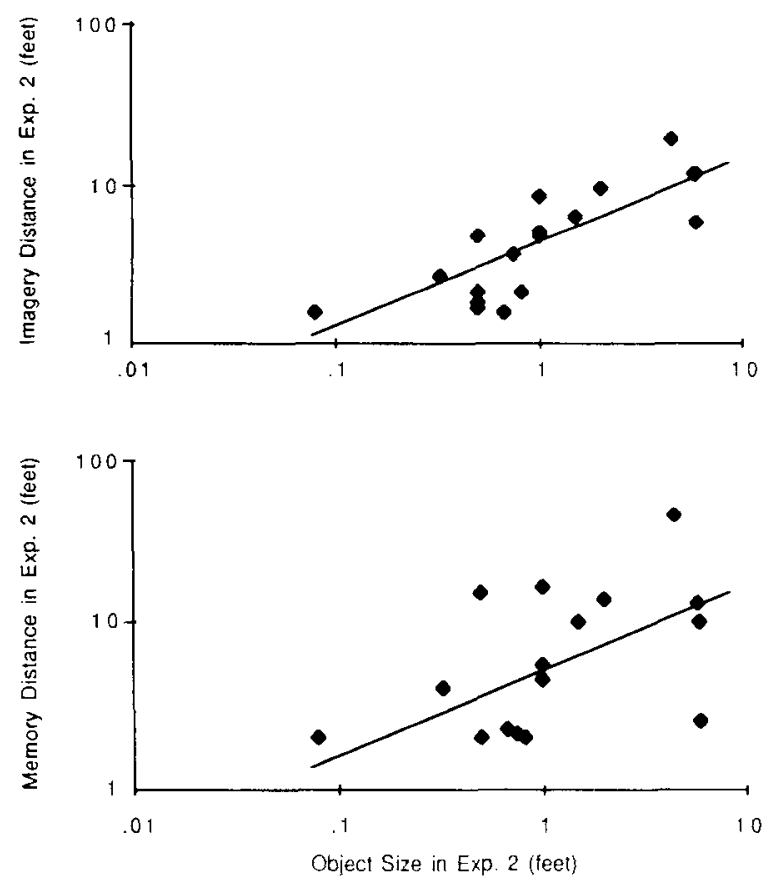

Figure 1. Median distance of imaged and remembered objects as a function of object size in Experiment 2 in logarithmic coordinates. The top panel shows distance of imaged objects; the bottom panel shows distance of remembered objects.

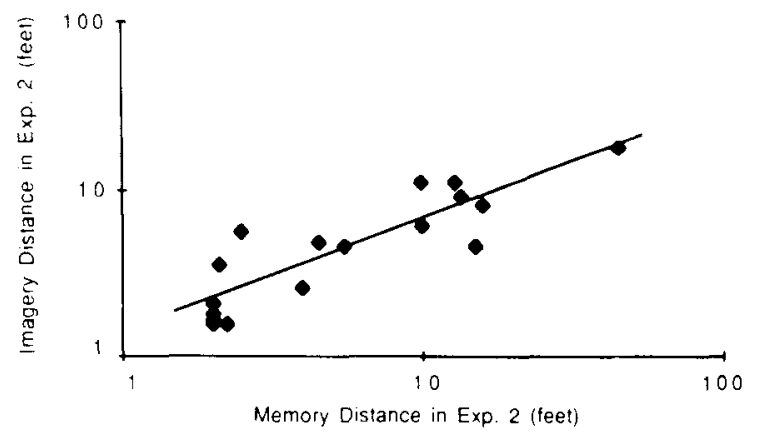

Figure 2. Median imaged distance as a function of median remembered distance in Experiment 2 in logarithmic coordinates.

distance estimates of different objects. The main effect for instruction was nonsignificant; that is, no difference was obtained in the distance estimates obtained under the imagery and memory instructions [quasi $F(2,34)=1.2$, $p>05]$. Not surprisingly, object size was highly significant, with larger objects being placed at greater distances $[F(17,306)=11.20, p<.001]$. The instruction $X$ object size interaction was nonsignificant $[F(17,306)$ $=1.12, p>.05$ ].

The subjects in the sorting group produced 81 separate stacks. The number of cards within each stack varied from 1 to 11 , and the number of stacks per subject varied from 3 to 9 . Subjects did not significantly discriminate between imagery and memory contexts $\left[\chi^{2}(80)=43.14\right.$, $p>.05]$. Subjects' professed criteria for sorting was based primarily on the similarity of physical setting (e.g., in a room, by a road, in a barnyard) for both the imagery and memory conditions. The only setting that was found in one list and not the other was "floating in space," which occurred in 3 (out of 180) imagery contexts.

Overall, the results are quite consistent with those from Experiment 1 . The distance portrayed in an image of a familiar object of stated size and the remembered distance of that object are very similar. Imaged and remembered distances are related to stated size by power functions with exponents less than 1 . However, in the case of familiar objects, there is considerable scatter around the function. The similarity of contexts, as well as the similarity of distances, further suggests that imagery and memory instructions may result in activation of similar cognitive processes or mechanisms.

\section{EXPERIMENT 3}

The distance estimates reported in the first two experiments highlight the inapplicability of the SDIH to distance estimation in untransformed images and nonimaginal memories. The similarity of the distance estimates for the imagery and the memory conditions of Experiments 1 and 2, along with the similarity of contexts found in Experiment 2 , is consistent with the notion that the cognitive processes underlying these judgments are highly similar, and may even be identical. However, the power functions 
obtained may have arisen from a more general strategy used by subjects in estimating distance, rather than from any intrinsic qualities of imagery or memory. To address this possibility, it was necessary to examine distance estimation in other situations. If estimates of physical distance based on perception of physical objects result in exponents similar to those obtained in Experiments 1 and 2 , then those earlier results may have arisen from a distance estimation process per se, and not from any special properties attributable to imagery or memory. If, however, the perceptual estimates are related to the physical distances in some other way, then previous results would not be due to estimation strategies and may be assumed to reflect properties of the memory or image.

To increase the range beyond that permitted by the size of the laboratory, the stimuli were chosen from an outdoor setting. To this end, a route around the campus was laid out from which objects of varying distance could be seen. The subjects walked the route and were stopped periodically to estimate the distance from where they stood to some specified object. This estimate was recorded, and the subject was escorted to the next stop.

\section{Method}

Subjects. The subjects consisted of 26 Dartmouth College undergraduates who received extra credit in an introductory psychology course.

Materials. The stimuli were 16 objects on the Dartmouth campus, including trees, buildings, newspaper boxes, benches, traffic signs, and trash cans (see Table 4). They were preselected so as to cover a wide range of distances (1 to $995 \mathrm{ft}$ ). No objects were moved about in the environment, and none was put there for the purpose of the experiment. The stimuli were located along a route that meandered through the campus.

General procedure. The subjects were run in five groups, but each subject worked individually. At the start of the experiment, the subjects were each provided with a sheet containing 16 blanks. They were instructed to record their distance estimates in the appropriate blanks. The subjects were then escorted around the route, pausing at the appropriate locations so that distance estimates could be made. The subjects were allowed as much time as they desired to make their estimates.

Table 4

Stimuli for Experiment 3

\begin{tabular}{lc}
\hline \multicolumn{1}{c}{ Object } & Distance (in feet) \\
\hline Trash can & 1 \\
Sewer grate & 3.5 \\
Road sign & 7.5 \\
Street lamp & 12 \\
Doorway & 29.5 \\
Tree & 40 \\
Street lamp & 72 \\
Tree & 97 \\
Park bench & 100 \\
Road sign & 111.5 \\
Trash can & 195 \\
Road sign & 242 \\
Building & 423 \\
Postal box & 520 \\
Newspaper box & 707 \\
Building & 995 \\
\hline
\end{tabular}

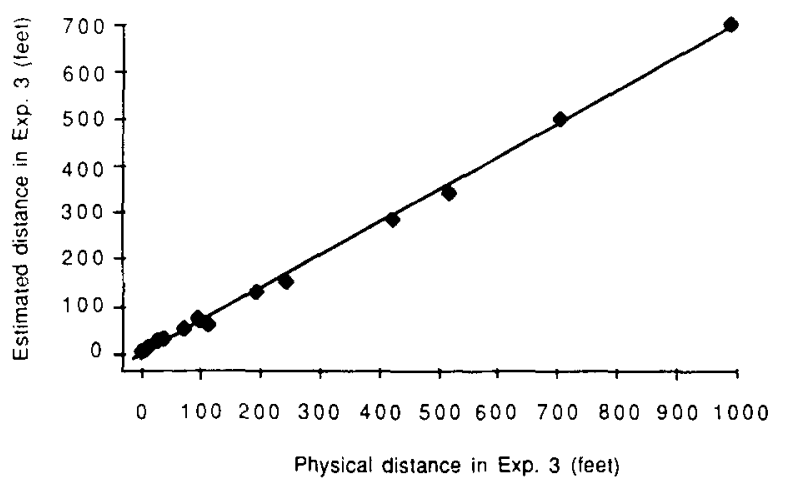

Figure 3. Median estimated distance as a function of physical distance in Experiment 3 in linear coordinates.

\section{Results and Discussion}

A median distance estimate was computed for each object. Estimated distance was significantly and strongly correlated with physical distance $(r=.999)$. These estimated distances are plotted against the appropriate physical distances in Figure 3 in linear coordinates. Least-squares regression yielded a $y$-intercept of -3.64 and a slope of $.697\left(r^{2}=.998\right)$.

The relationship between estimated and physical distance for perceived objects is strikingly linear. This is consistent with previous studies, which have found the exponent to be 1 or slightly less than 1 for perceived distance along the ground (Da Silva, 1985; Wagner, 1985; Wiest \& Bell, 1985). Linear functions (exponents close to 1) are also found when subjects estimate from memory the distances between buildings in a familiar campus environment (Baird, Merrill, \& Tannenbaum, 1979; Sherman, Croxton, \& Giovanatto, 1979). This linear trend differs substantially from the power functions for imaged and recalled distance.

The subjects in this experiment, unlike in the previous experiments, were not told the sizes of the objects they were viewing. We do not believe this is critical, since all the objects were commonly found on the campus, and thus were familiar to the subjects. It is also unlikely that the distances to the specific objects would have been judged by the subjects prior to the experiment, so the likelihood of any explicit prior judgment, expressed in feet and inches, is miniscule. Overall, these results suggest that the low exponents in previous experiments are due to properties of the image and/or memory representation, and are not due to strategies of distance estimation.

\section{GENERAL DISCUSSION}

The data of Experiments 1 and 2 do not agree with the SDIH defined by Equation 1. For both familiar objects and nondescript rods, the relation between estimated distance and known size is positive but nonlinear. This is not an artifact of the distance estimation process, as perceived distance is typically a linear function or a power function with an exponent near 1 . Thus, previous research showing a linear relationship between stated object size 
and imaged overflow distance (Kosslyn, 1980) cannot be due simply to subjects' recalling the usual distance of objects when they are asked to report the distance to an object at the overflow point of the visual field. Rather, some additional size-distance scaling is required. The positively monotonic function relating size and distance does suggest, however, that metric information is present even in untransformed representations.

Since rods of the size employed in Experiment 1 were probably never seen by subjects, distance estimates might have depended on recall of familiar objects that matched the sizes of the rods. Had this occurred, the function relating the size of the rod to imaged distance would have been as variable as the function relating the size of familiar objects to imaged distance. Unlike the case of familiar objects, however, distance estimates for the unfamiliar rods demonstrated minimal variability. It is more plausible that judgment of an individual rod is based on a statistical aggregate (mean or median) of numerous representations of objects of the same familiar size. In either event, our findings would not be expected if scale properties of size and distance were missing from the recalled or imaged representation prior to any scale transformation.

Those objects that we expect to be seen most often at distances inappropriate for their physical size (according to Equation 1) are indeed reported at their familiar distances. For example, the crow and the bird's nest are relatively small objects that are seldom seen up close. The fact that subjects report these stimuli as relatively distant distorts any monotonic relationship that otherwise exists between distance and size. The same holds true for the various people used as stimuli: the actor and policeman are recalled and imaged at greater distances than are the friend and store clerk. Familiarity with the typical perceived distances of objects tends to distort the underlying relationship for imaged and remembered distance, and, in the absence of familiarity, less distortion occurs.

This effect of familiarity is consistent with the idea that some aspects of imagery are cognitively penetrable, that is, affected by subjects' beliefs, expectations, and knowledge (Pylyshyn, 1981). We found no differences in distance estimates between instructions to image and instructions to remember, consistent with Anderson's (1978) suggestion that coded episodes could be described equally well as images or as memories, and also consistent with views suggesting functional equivalence at some level of memory and imagery (see Marschark, Richman, Yuille, \& Hunt, 1987)

The findings support the notion that the memory representations used to create visual images contain contextual information about the metric sizes and distances of objects. Although the SDIH for untransformed images was not verified in the strict sense, there clearly was a positive relationship between the size and distance of an object represented in memory; therefore, metric information is already inherent in the initial formation of an image. In order for the SDIH to hold true, it appears that additional size-distance scaling of the inherent metric in- formation may be necessary (see Hubbard \& Baird, 1988).

\section{REFERENCES}

ANDERSON, J. R. (1978). Arguments concerning representations for mental imagery. Psychological Review, 85, 249-277.

BAIRD, J. C. (1963). Retinal and assumed size cues as determinants of size and distance perception. Joumal of Experimental Psychology, 66, 155-162

BAIRD, J. C. (1970). Psychophysical analysis of visual space. New York: Pergamon Press.

Baird, J. C., Green, D. M., Luce, R. D. (1980). Variability and sequential effects in cross-modality matching of area and loudness. Journal of Experimental Psychology: Human Perception \& Performance, 6, 277-289.

Baird, J. C., Merrlll, A. A., \& Tannenbaum, J. (1979). Cognitive representation of spatial relations: II. A familiar environment. Journal of Experimental Psychology: General, 108, 92-98.

BAIRD, J. C., NomA, E. (1978). Fundamentals of scaling and psychophysics. New York: Wiley.

Coltheart, M. (1969a). Effects of two kinds of distance information on visual judgments of absolute size. Nature, 221, 338.

Coltheart, M. (1969b). The influence of haptic size information upon visual judgments of absolute distance. Perception \& Psychophysics, 5, 143-144.

COLtheart, M. (1970). The effect of verbal size information upon visual judgments of absolute distance. Perception \& Psychophysics, 9 , 222-223.

DA Silva, J. A. (1985). Scales for perceived egocentric distance in a large open field: Comparison of three psychophysical methods. American Journal of Psychology, 98, 119-144.

Epstein, W., PARK, J., \& CASEY, A. (1961). The current status of the size-distance invariance hypothesis. Psychological Bulletin, 58, 491-514

Farah, M. (1985). Psychophysical evidence for a shared representational medium for mental images and percepts. Joumal of Experimental Psychology: General, 114, 91-103.

Finke, R. A., \& ShePARD, R. N. (1986). Visual functions of mental imagery. In K. R. Boff, L. Kaufman, \& J. P. Thomas (Eds.), Handbook of perception and human performance. Vol. 2: Cognitive processes and performance (pp. 37.1-37.55). New York: Wiley.

FitzPatrick, V., Pasnak, R., \& TYer, Z. (1982). The effect of familiar size at familiar distances. Perception, 11, 85-91.

Hastorf, A. H. (1950). The influence of suggestion on the relationship between stimulus size and perceived distance. Joumal of Psychology, 29, 195-217.

HubBaRD, T. L., \& BaiRD, J. C. (1988). Overflow, first-sight, and vanishing point distances in visual imagery. Journal of Experimental Psychology: Learning, Memory, \& Cognition, 14, 641-649.

ItTelson, I. H. (1951). Size as a cue to distance: Static localization. American Journal of Psychology, 64, 54-67.

KossLyn, S. M. (1978). Measuring the visual angle of the mind's eye. Cognitive Psychology, 10, 356-389.

Kosslyn, S. M. (1980). Image and mind. Cambridge, MA: Harvard University Press.

Marschark, M., Richman, C. L., Yuille, J. C., \& Hunt, R. R. (1987). The role of imagery in mernory: On shared and distinctive information. Psychological Bulletin, 102, 28-41.

Ono, H. (1969). Apparent distance as a function of apparent size. Journal of Experimental Psychology, 79, 109-115.

Park, J. N., \& Michaelson, G. J. (1974). Distance judgments under different size-information conditions. Perception \& Psychophysics, $15,57-60$.

Pylyshyn, Z. W. (1981). The imagery debate: Analog media versus tacit knowledge. Psychological Review, 87, 16-45.

SEDGWICK, H. A. (1986). Space perception. In K. R. Boff, L. Kaufman, \& J. P. Thomas (Eds.), Handbook of perception and human performance. Vol. 1: Sensory processes and perception (pp. 21.121.57). New York: Wiley 
SEgAL, S. J., Fusella, V. (1970). Influence of imaged pictures and sounds on detection of visual and auditory signals. Journal of Experimental Psychology, 83, 458-464.

Shepard, R. N., Podgorny, P. (1978). Cognitive processes that resemble perceptual processes. In W. K. Estes (Ed.), Handbook of learming and cognitive processes (Vol. 5, pp. 189-237). New York: Halsted Press.

Sherman, R. C., Croxton, J., \& Govanatto, J. (1979). Investigating cognitive representations of spatial relationships. Environment \& Behavior, 11, 209-216.

WAGNer, M. (1985). The metric of visual space. Perception \& Psychophysics, 38, 483-495.
Weber, R. J., \& Malmstrom, F. V. (1979). Measuring the size of mental images. Joumal of Experimental Psychology: Human Perception \& Performance, 5, 1-12.

WIEST, W. M., BeLL, B. (1985). Steven's exponent for psychophysical scaling of perceived, remembered, and inferred distance. Psychological Bulletin, 98, 457-470.

(Manuscript received July 21, 1987;

revision accepted for publication April 1, 1988.) 